\section{Incisor reduction: a provisional aesthetic technique for traumatised teeth}

\author{
L. J. Darby' and A. C. O'Connell ${ }^{2}$
}

VERIFIABLE CPD PAPER
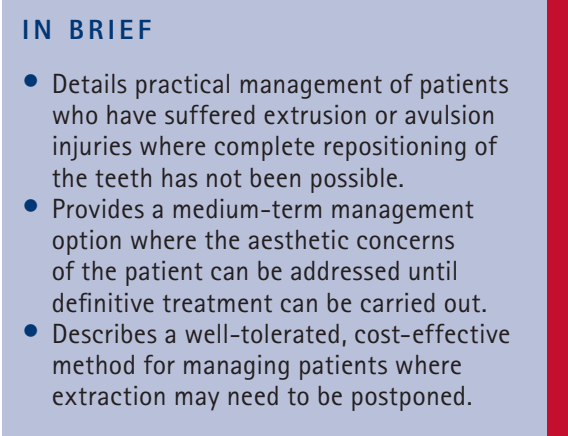

Patients in the mixed dentition who have suffered severe extrusion or avulsion injuries often present with difficult treatment decisions, especially when the initial emergency care has been compromised. Here we describe a well-tolerated, aesthetically acceptable and conservative method for treating such patients until a definitive treatment plan is possible.

\section{INTRODUCTION}

The most successful outcomes of an avulsion or extrusion injury occur where the tooth is returned to the original position as soon as possible following trauma. ${ }^{1,2,3}$ Delays in seeking treatment, poor cooperation and severity of the traumatic injuries can result in incomplete repositioning of teeth at the time of injury. ${ }^{4}$ Once emergency care has been provided, decisions must be made with regard to the medium- and long-term treatment of the patient, including planning for pre-existing skeletal and dento-alveolar discrepancies. In children in the mixed dentition the priority is to maintain a stable dental condition that is acceptable to the patient and that does not impair future treatment.

Options for improving aesthetics include restorative or orthodontic treatment, autotransplantation, ${ }^{5}$ decoronation ${ }^{6}$ or extraction and prosthetic replacement, ${ }^{7,8}$ Treatment planning can be complicated by factors such as the mixed dentition, individual rate of growth, poor cooperation of the child and dental motivation of the family. Orthodontic therapy may not be advised in mixed dentition if teeth are

\footnotetext{
'Postgraduate orthodontic student, Cork University Dental School and Hospital, Wilton, Cork, Ireland; ${ }^{2}$ Consultant/Senior Lecturer in Paediatric Dentistry, Division of Public and Child Dental Health, Dublin Dental School and Hospital, Trinity College Dublin, Lincoln Place, Dublin 2, Ireland

${ }^{*}$ Correspondence to: Anne 0'Connell

Email: anne.oconnell@tcd.ie
}

\section{Refereed Paper}

Accepted 15 September 2010

DOI: 10.1038/sj.bdj.2010.1083

${ }^{\bullet}$ British Dental Journal 2010; 209: 553-556 partially erupted or have recently been traumatised. ${ }^{8}$ Extraction of the malpositioned teeth with poor long-term prognosis may result in early loss of bone that could compromise future implant therapy. ${ }^{7}$ In addition, wearing a removable prosthesis may be unacceptable aesthetically or may not be possible due to poor behaviour or cooperation in some young patients. We describe the conservative treatment of recontouring the traumatised teeth. This is a cost-effective, well-tolerated and inexpensive option of maintaining the teeth until such time as it is suitable to undertake a definitive treatment plan.

\section{CASE 1}

A healthy 91/2-year-old girl fell from a height and traumatised the upper anterior teeth. Following the initial trauma the patient attended her own general dentist. She had avulsed her upper right permanent central incisor, and her upper right permanent lateral incisor had been severely extruded. The upper right primary canine was also avulsed. The permanent right central incisor was replanted after one hour of extra-oral dry time (EODT) and 30 minutes storage in milk. The permanent lateral incisor was also repositioned at this time. The teeth were then splinted using a composite splint (Fig. 1a). She attended the Accident and Emergency Department of the Dublin Dental Hospital the following day. At this time the incisors were further repositioned digitally and resplinted using a flexible composite-wire splint (Fig. 1b). Pulp extirpation of both incisors was carried out as recommended by the guidelines ${ }^{3}$ and

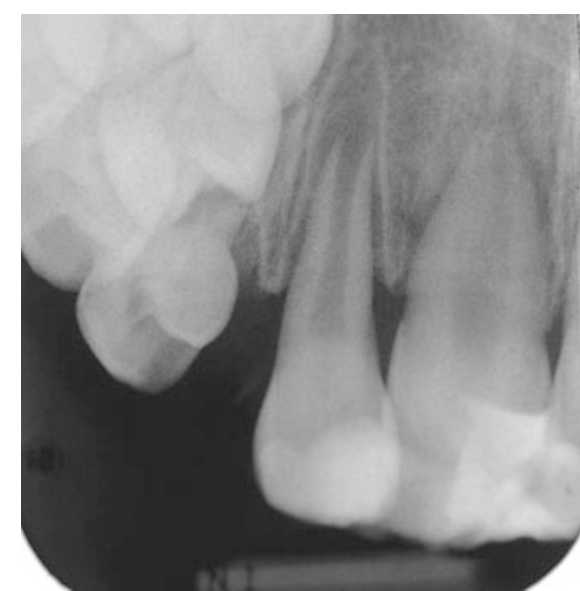

Fig. 1a Case 1: Periapical radiograph of traumatised upper right permanent central and lateral incisorsat time of injury showing upper right lateral incisor not fully repositioned into socket

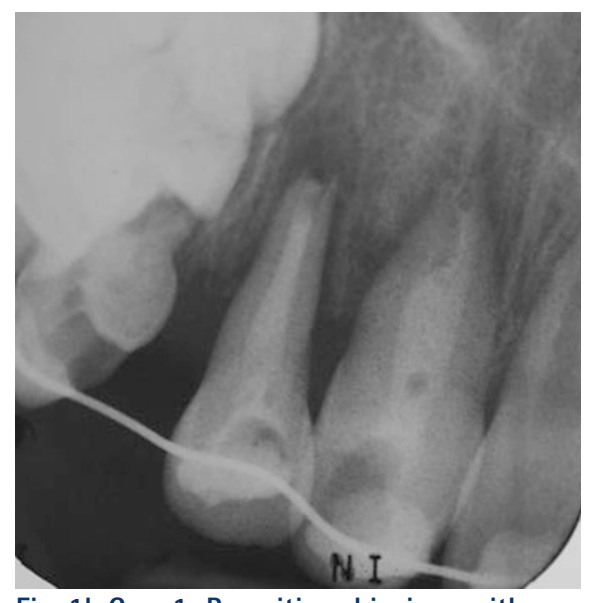

Fig. 1b Case 1: Repositioned incisors with wire-splint in situ and first stage endodontic treatment

non-setting calcium hydroxide (UltraCal, Ultradent, South Jordan, UT) was placed in the canals (Fig. 1c). After three changes of $\mathrm{CaOH}$ over 14 months, obturation of these 


\section{PRACTICE}

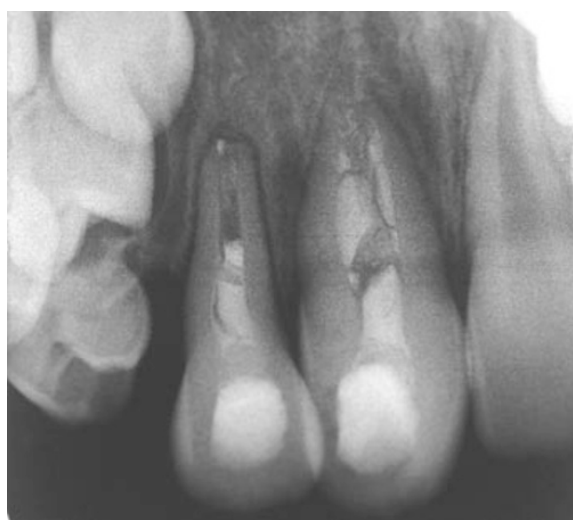

Fig. 1c Case 1: One year post trauma demonstrating healing. Note also the incisal edge discrepancy between upper right and left central incisors

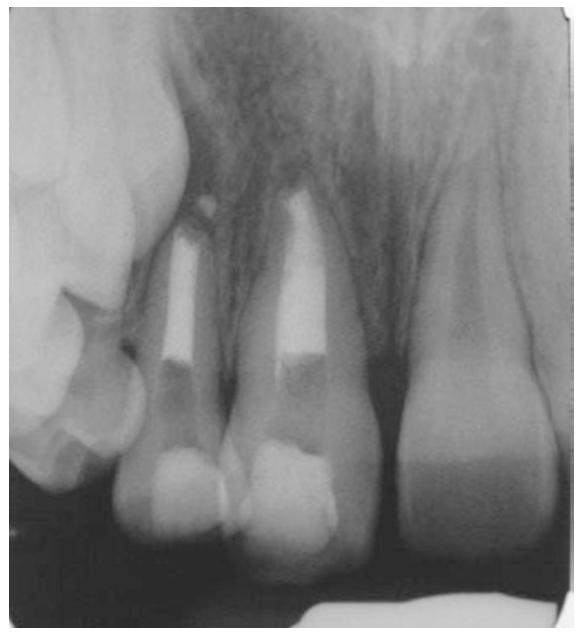

Fig. 1d Case 1: 19 months post trauma with obturation of upper right central and lateral incisors with MTA and GP

teeth was completed with mineral trioxide aggregate (MTA, ProRoot MTA, Dentsply Tulsa Dental, Tulsa, OK) and thermoplastic gutta percha technique (Obtura, Unitek, Monrovia, CA) and sealed using composite resin restoration (Fig. 1d).

The patient and parent were aware of the poor long term prognosis of both of these teeth. An orthodontic opinion suggested that any orthodontic treatment be delayed to allow the permanent canine to be guided into position (Fig. 1d). The poor aesthetics of the incomplete repositioned teeth had become a concern to the patient within months of the trauma (Figs 2a, 2b). Extraction of right lateral incisor and provision of a removable prosthesis was not an acceptable option for the patient and would have allowed the permanent canine to move mesially. Tooth recontouring was suggested as a reasonable interim aesthetic solution. The reduction was marked on both teeth with an alcohol pen and incisal

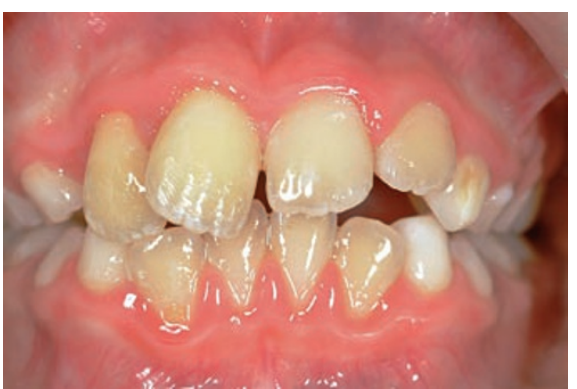

Fig. 2a Case 1: Clinical photographs of traumatised upper right central and lateral incisors. The aesthetic appearance is poor due to incisal discrepancy of the maxillary incisors

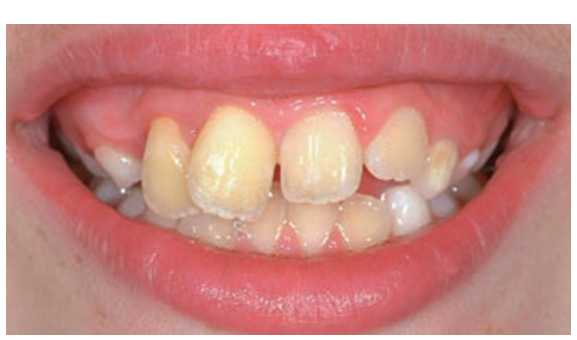

Fig. $2 b$ Case 1: Clinical appearance of smile pre-contouring

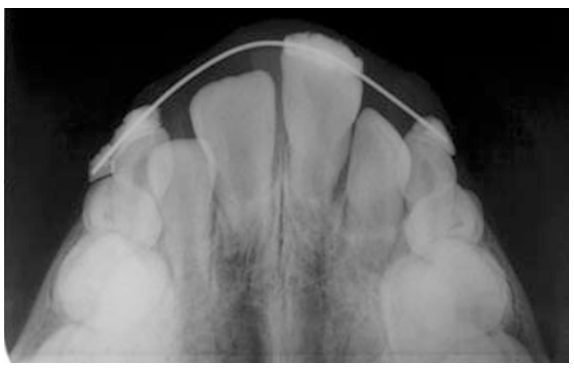

Fig. 3a Case 2: Anterior occlusal radiograph demonstrating traumatised upper right central and lateral incisors, with incomplete positioning of upper left central incisor

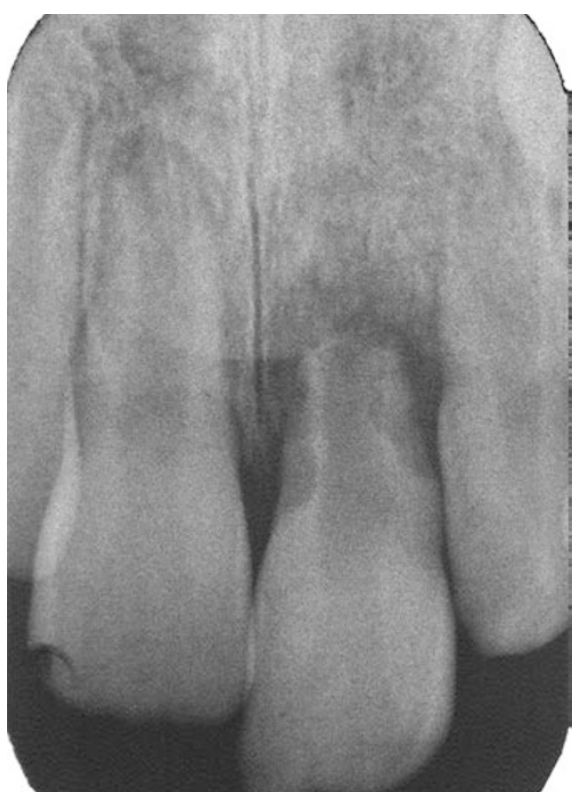

Fig. 3b Case 2: Periapical radiograph 2 months post trauma showing resorption of upper left central incisor and incisal level discrepancy between upper central incisors

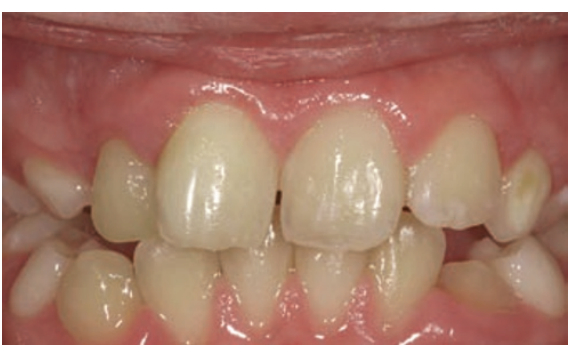

Fig. 2c Case 1: Incisal and distal reduction of incisors

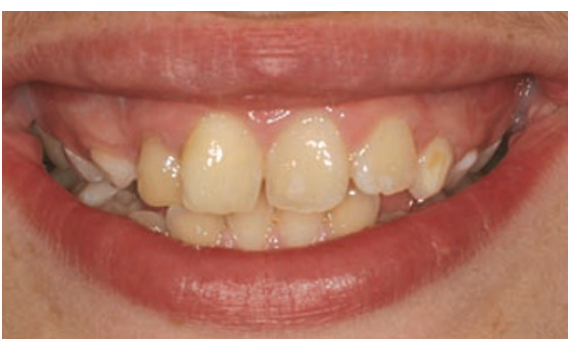

Fig. $2 \mathrm{~d}$ Case 1: Clinical appearance of smile approximately $\mathbf{2 8}$ months post trauma demonstrating stability of recontoured upper right permanent central and lateral incisors

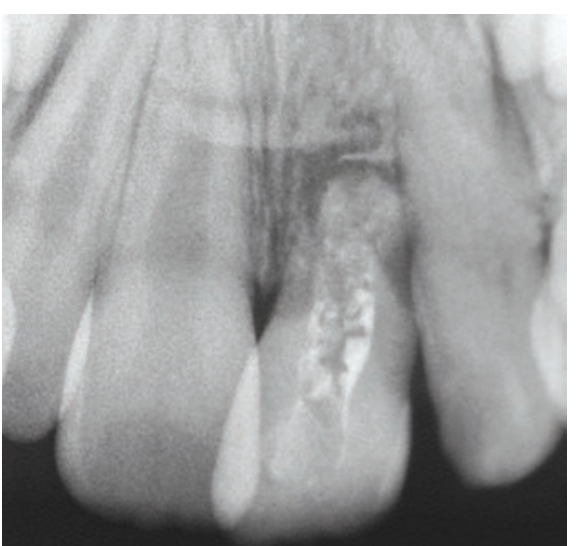

Fig. 3c Case 2: Periapical radiograph taken 18 months post trauma with calcium hydroxide for control of resorption. The non-setting calcium hydroxide had 'washed out' since the previous visit and the tooth was redressed at this visit

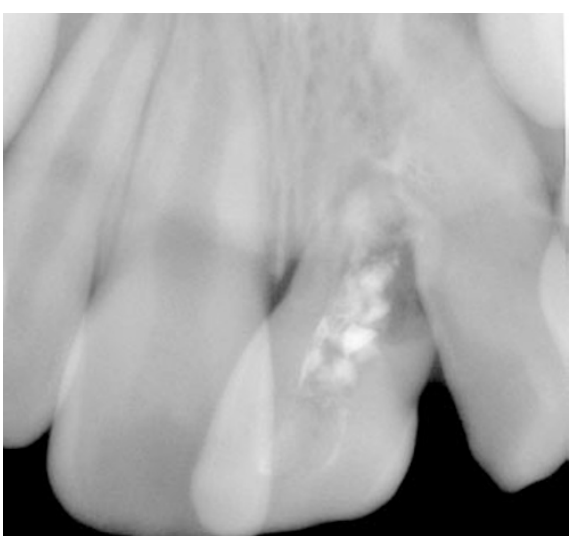

Fig. 3d Case 2: Periapical radiograph taken 30 months post trauma demonstrating continued resorption. The non-setting calcium hydroxide had 'washed out' since the previous visit and the tooth was redressed at this visit 
and distal reduction was carried out using a diamond bur. Both patient and parents were very pleased with the result (Fig. 2c,) which has remained stable for two years (Fig. 2d). A decision on the timing of removal of the lateral incisor will be made by the orthodontist. Currently it is guiding the permanent canine into a favourable position while avoiding an edentulous space anteriorly.

\section{CASE 2}

A healthy 8-year-old girl presented at the Accident and Emergency Department of the Dublin Dental Hospital, having fallen in her kitchen two hours previously. She avulsed her upper left permanent central incisor which subsequently had an EODT of 40 minutes followed by one and a half hours' storage in milk. She also suffered laceration of her upper lip, a mild ( $<3 \mathrm{~mm}$ ) intrusive luxation of her upper right permanent incisor and subluxation of her upper left permanent lateral incisor. She and her parents were made aware of the poor prognosis of upper left central incisor due to the nature and severity of the trauma and the delayed treatment, but the parents requested replantation of the avulsed tooth. At the emergency appointment the upper left permanent central incisor was replanted and splinted with a functional composite-wire splint. It was not possible to fully reposition the tooth or to reduce the alveolar fracture at this time due to poor co-operation of the child (Fig. 3a). Two weeks later the splint was removed, but pulp extirpation of the left central incisor could not be carried out due to behaviour problems. The intruded right central incisor appeared to have re-erupted, but there was a discrepancy of $6 \mathrm{~mm}$ between the incisal edges of the central incisors (Fig. 4a, Fig. 5a). Although pulp extirpation was advised to prevent inflammatory root resorption, it was not performed due to the child's behaviour and reluctance of the parents to consent to the proposed treatment at this time. The patient was referred to a paediatric dentist for behavioural modification to facilitate any further treatment. Over the next three weeks further eruption of the right central incisor reduced the discrepancy between the incisal edges to $4.5 \mathrm{~mm}$. Two months post trauma external inflammatory resorption of the left central incisor was diagnosed radiographically

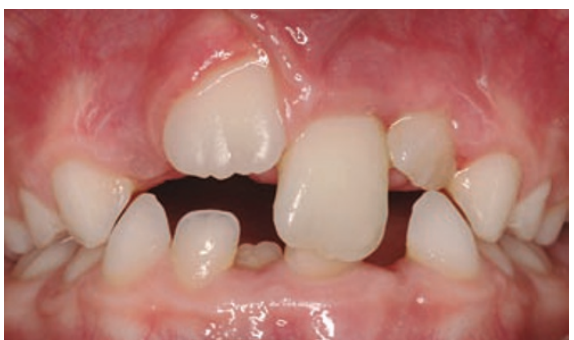

Fig. 4a Case 2: Clinical photographs of traumatised upper right and left central incisors. Discrepancy of gingival margin and incisal edge of the traumatised teeth 1 month following trauma

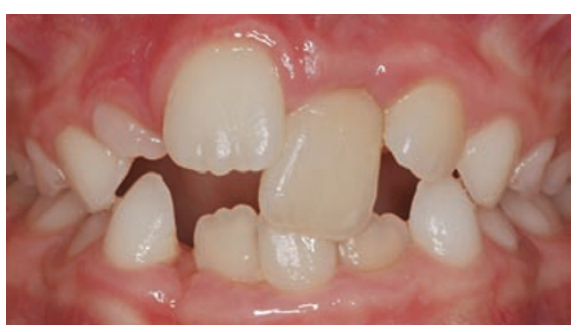

Fig. 4b Case 2: A decrease in incisal discrepancy 11 months post trauma due to continued eruption of anterior teeth

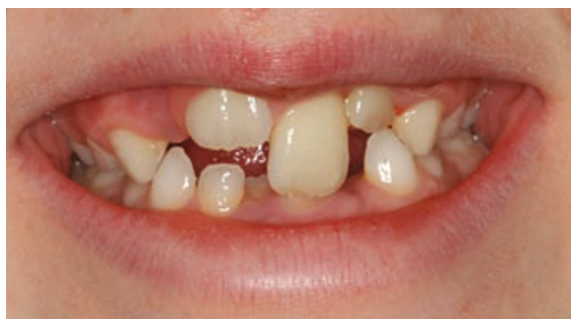

Fig. 5a Case 2: Anterior appearance one month post trauma

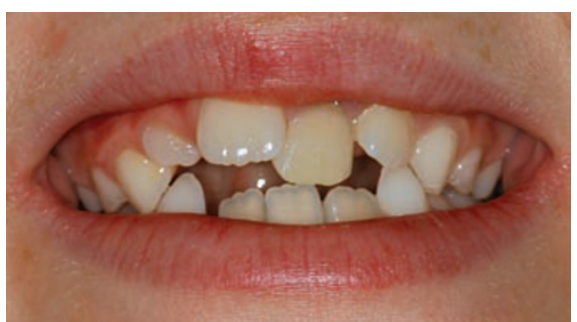

Fig. 5b Case 2: Appearance 14 months post trauma showing recontouring of upper left central incisor and further eruption of upper right central incisor

(Fig. 3b). The parents consented to pulp extirpation and calcium hydroxide dressing (Ultracal, Ultradent, South Jordan, UT) to resolve any infection and maintain bone in the area as a medium term goal. The patient was reviewed periodically and nonsetting calcium hydroxide changed at three monthly intervals.

Three months post trauma, the upper right central incisor continued to erupt and showed continued root development. The upper left central incisor continued to

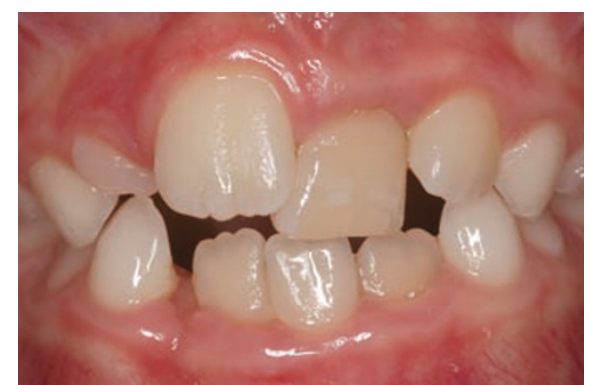

Fig. 4c Case 2: Initial incisal reduction of upper left central incisor

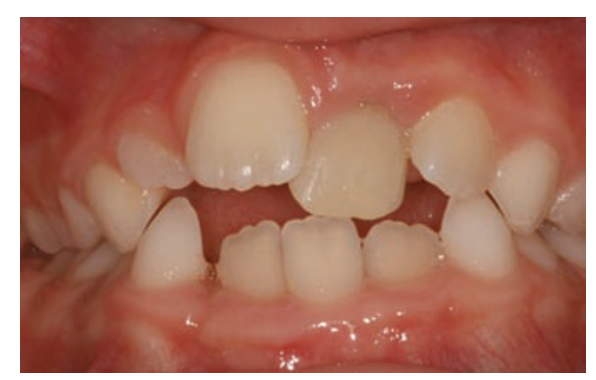

Fig. 4d Case 2: Clinical appearance after further recontouring. Note the palatal and mesial movement of upper left central incisor

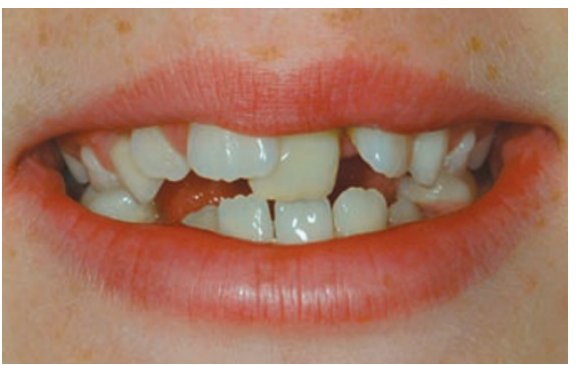

Fig. 5c Case 2: Mesial and palatal movement of upper left central incisor $\mathbf{3 0}$ months post trauma

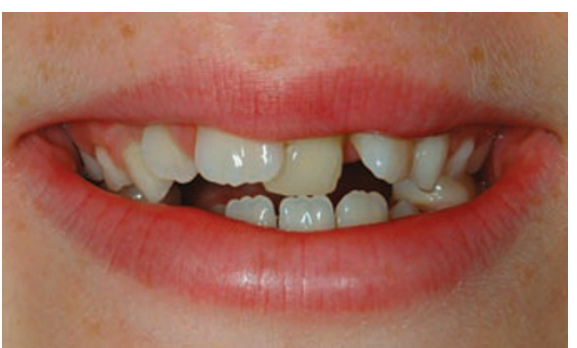

Fig. 5d Case 2: Additional recontouring of upper left central incisor

exhibit Grade I mobility, and showed signs of replacement resorption. The incisal edge discrepancy was reduced from $4 \mathrm{~mm}$ to $3.5 \mathrm{~mm}$ at six months post trauma and further to $3 \mathrm{~mm}$ at ten months after the injury. This appears to be due to continued eruption of the right central incisor, but at one year post trauma the discrepancy remained at $3 \mathrm{~mm}$ (Fig. 4b). Replacement resorption of the left central incisor progressed and prevented the continued vertical growth of the alveolus, and the 
tooth was now more mesially and palatally placed than its antimere (Fig. 3c, 3d). Once again extraction and replacement of the tooth was declined but an aesthetic improvement was requested by the parents (Figs. 5c). Recontouring of the left central incisor was approved and the tooth was reduced using diamond burs as previously described (Figs. 4c, 4d, 5b). Eruption of the adjacent lateral incisors and development of the alveolus continued but the position of the left central incisor continued to deteriorate (Fig. 5c). A further reduction was performed (Fig. 5d) and both parents and child are very happy with the result.

Poor cooperation of the child and continued reluctance by the parents to agree to intervention for the traumatised tooth have complicated future treatment for the child. All are aware that orthodontic therapy will be required to align teeth correctly and restoration of the space following loss of the left central incisor may be compromised now that a dento-alveolar defect is present. This case highlights the difficulty of balancing appropriate dental care with parents' expectations and wishes. As dentists, we recognise the midline shift, tooth migration and angulation and the discrepancy in the gingival level, yet the parents and child are very happy with the smile at this time (Figs. 5a-5d).

\section{DISCUSSION}

The importance of an aesthetic smile for self-esteem in young patients cannot be underestimated. ${ }^{9}$ The treatment options include joint orthodontic and restorative management, autotransplantation, ${ }^{5}$ decoronation $^{6}$ and extraction. Management of trauma in the mixed dentition can be complicated, especially where initial treatment has been compromised. Treatment planning involves realistic evaluation of the prognosis of the traumatised teeth, aesthetics and the eventual orthodontic management of any malocclusion. The most appropriate treatment for the patient can be further complicated where complete repositioning of the traumatised tooth has not been possible, especially in the growing child. Regardless of the success of the pulp therapy, the aesthetics of the traumatised teeth are often paramount for the child patient and parent. Tooth recontouring is a valuable and economical provisional treatment option for patients in the mixed dentition who have suffered incomplete repositioning of anterior teeth following an avulsion or extrusive injury, especially when the tooth is unlikely to be retained long term. It provides an aesthetic option for the compromised tooth until such time as a definitive treatment plan is agreed. It is advantageous in cases where a removable appliance is contra-indicated. Maintaining such a traumatised tooth may be beneficial where extraction might compromise future implant prognosis. It is an acceptable, inexpensive aesthetic option for the management of incompletely repositioned teeth. The technique described here is recommended for cases with incompletely repositioned non-vital teeth. Caution must be used if this technique is to be used in vital teeth because of the risk of dentine exposure and damage to a healthy pulp. Knowledge of tooth morphology is also of paramount importance to achieve a good aesthetic result. However, it is rarely a long-term treatment option. Achieving an aesthetic improvement is the aim of this treatment technique, although this may not always result in an ideal aesthetic appearance.

In the first case, the loss of the avulsed tooth is likely due to the unfavourable EODT, however the orthodontist advised maintenance of the the traumatised teeth in the medium term to guide correct positioning of the permanent canine. In the second case the lack of parental consent and patient cooperation limited the treatment options available. Failure to extirpate pulp in a timely manner results in the development of inflammatory resorption. ${ }^{3}$ Eventual treatment with $\mathrm{CaOH}$ will control inflammatory resorption but the compromised post-trauma treatment regimen increased the risk of replacement resorption and eventual loss of this tooth. ${ }^{8}$ It is obvious that patient/parent needs must supercede dental outcomes as long as all decisions are informed. The parents accepted that they had agreed to compromised treatment but are very happy with the outcome.
With correct case selection, incisal reduction can be a valuable conservative and well-tolerated technique for provisional treatment of incompletely repositioned teeth post-trauma in patients in the mixed dentition. The degree of recontouring must anticipate the continued eruption of the adjacent teeth that occurs due to normal growth and development in a child in the mixed dentition. The level of the smile line is also important as this treatment will not alter the gingival height. The reduction of other teeth should not occur unless agreed within the definitive treatment plan. Parents must be aware that there are limitations to this treatment option, and further treatment will be required for superior aesthetics, particularly where there are coexisting orthodontic treatment needs.

\section{CONCLUSION}

Tooth recontouring is an economical technique to consider when a patient or parent finds other treatment options unacceptable. It is also a useful interim measure while awaiting further dental development before appropriate interdisciplinary care can be undertaken. This provisional treatment modality will satisfy aesthetic demand and also can maintain space and alveolar bone until a definitive plan can be formulated.

1. Flores M T, Andersson L, Andreasen J 0 et al. Guidelines for the management of traumatic dental injuries. I. Fractures and luxations of permanent teeth. Dent Traumato/ 2007; 23: 66-71.

2. Flores M T, Andersson L, Andreasen $J 0$ et al. Guidelines for the management of traumatic dental injuries. II. Avulsion of permanent teeth. Dent Traumato/ 2007; 23: 130-136.

3. Dental trauma guide. http://www.dentaltraumaguide.org [accessed 15 September 2009].

4. Humphreys K, Al Badri S, Kinirons M et al. Factors affecting outcomes of traumatically extruded permanent teeth in children. Pediatr Dent 2003; 25: 475-478.

5. Andreasen J O, Paulsen H U, Yu Z, Ahlquist R, Bayer T, Schwartz 0. A long term study of 370 autotransplanted premolars. Part I. Surgical procedures and standardized techniques for monitoring healing. Eur J Orthod 1990; 12: 3-13.

6. Malmgren B. Decoronation: how, why, and when? $J$ Calif Dent Assoc 2000; 28: 846-854.

7. Schwartz-Arad D, Levin L, Ashkenazi M. Treatment options of untreatable traumatized anterior maxillary teeth for future use of dental implantation. Implant Dent 2004; 13: 11-19.

8. Andreasen J O, Andreasen F M, Andersson L. Textbook and color atlas of traumatic injuries to the teeth. 4th ed. Oxford: Blackwell Munksgaard, 2007.

9. Marques L S, Filogônio C A, Filogônio C B et al. Aesthetic impact of malocclusion in the daily living of Brazilian adolescents. J Orthod 2009: 36: 152-159. 